\title{
A new tealliocaridid crustacean from the Late Carboniferous of North China and its biogeographic implications
}

Qiang Yang, Pierre Gueriau, Sylvain Charbonnier, Dong Ren, and Olivier Béthoux Acta Palaeontologica Polonica 63 (1), 2018: 111-116 doi:https://doi.org/10.4202/app.00446.2017

A new tealliocaridid eumalacostracan is described from the Late Carboniferous Tupo Formation (Ningxia, China). Laevitealliocaris xiaheyanensis gen. et sp. nov. is represented by a single specimen, characterised by the possession of a short rostrum without dorsal spine, a short postcervical carina and only one weak branchial carina, both tuberculate, and a short sixth pleonal somite. This is the first unequivocal record of tealliocaridids outside Euramerica, which occurrence along the eastern inner margin of the Palaeotethys suggests that these crustaceans were more widely distributed than previously recognised, very likely extending to the whole intertropical area. The new occurrence demonstrates that tealliocaridids had strong dispersal capacities, interestingly challenging their affinities with peracarids, which today do not have free-living larvae, unlike decapod crustaceans.

Qiang Yang [yq11_1984@126.com], School of Life Sciences, Guangzhou University, 230 GuangZhou University City Outer Ring Road, Guangzhou 510006, PR China; College of Life Science, Capital Normal University, 105 Xisanhuanbeilu, Haidian District, Beijing 100048, China; State Key Laboratory of Biocontrol, Key Laboratory of Biodiversity Dynamics and Conservation of Guangdong Higher Education Institute, Ecology and Evolution, School of Life Sciences, Sun Yat-sen University, Guangzhou 510275, China. Pierre Gueriau [pierre.gueriau@synchrotron-soleil.fr] (corresponding author), IPANEMA, CNRS, ministère de la Culture, UVSQ, USR3461, Université Paris-Saclay, F-91192 Gif-sur-Yvette, France. Sylvain Charbonnier [scharbonnier@mnhn.fr] and Olivier Béthoux [obethoux@mnhn.fr ], Sorbonne Universités, UPMC Univ Paris 06, MNHN, CNRS, Centre de Recherche sur la Paléobiodiversité et les Paléoenvironnements (CR2P), Paris, France - Muséum National d'Histoire Naturelle, 57 rue Cuvier, CP38, F-75005 Paris, France. Dong Ren [rendong@mail.cnu.edu.cn] (corresponding author), College of Life Science, Capital Normal University, 105 Xisanhuanbeilu, Haidian District, Beijing 100048, China. 
This is an open-access article distributed under the terms of the Creative Commons

Attribution License (for details please see creativecommons.org), which permits unrestricted use, distribution, and reproduction in any medium, provided the original author and source are credited.

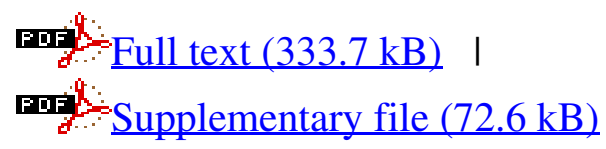

